02,01

\title{
Особенности нелинейного СВЧ-отклика многозонных сверхпроводников на основе ферроселенида натрия-калия $\left(\mathrm{Na}_{0.3} \mathrm{~K}_{0.7}\right)_{x} \mathrm{Fe}_{2-y} \mathrm{Se}_{2}$
}

\author{
(C) Е.Е. Пестов ${ }^{1,2}$, Ю.Н. Ноздрин ${ }^{1}$, А.И. Елькина ${ }^{1}$, Ю.С. Ерин ${ }^{1}$, М. Лю ${ }^{3}$, \\ А.И. Болталин ${ }^{3}$, И.В. Морозов ${ }^{3}$
}

${ }^{1}$ Институт фоизики микроструктур РАН, Нижний Новгород, Россия

${ }^{2}$ Нижегородский государственный университет им. Н.И. Лобачевского, Нижний Новгород, Россия

${ }^{3}$ Московский государственный университет им. М.В. Ломоносова, Москва, Россия

E-mail: pestov@ipmras.ru

С помощью метода нелинейной ближнепольной СВЧ-микроскопии были изучены температурные зависимости мощности третьей гармоники для монокристаллов ферроселенида натрия-калия $\left(\mathrm{Na}_{0.3} \mathrm{~K}_{0.7}\right)_{x} \mathrm{Fe}_{2-y} \mathrm{Se}_{2}$. Наряду с этим проведены измерения температурной зависимости магнитной восприимчивости на частоте $100 \mathrm{kHz}$ для данного соединения. Полученные экспериментальные результаты указывают на возможную двухщелевую структуру сверхпроводящего состояния $\left(\mathrm{Na}_{0.3} \mathrm{~K}_{0.7}\right)_{x} \mathrm{Fe}_{2-y} \mathrm{Se}_{2}$.

Работа частично поддержана РФФИ (грант № 18-02-00912 и грант № 18-42-520021), РНФ (грант № 15-12-10020) и Программой фундаментальных исследований Президиума РАН „Фундаментальные проблемы высокотемпературной сверхпроводимости“. В работе использовано оборудование ЦКП „Физика и технология микро- и наноструктур“.

DOI: 10.21883/FTT.2018.11.46649.27NN

\section{1. Введение}

Экспериментальное обнаружение многозонной структуры сверхпроводящего состояния в дибориде магния в 2001 г. [1] и пниктидах и халькогенидах железа в 2008 г. [2] привело к появлению теоретических и экспериментальных работ, в которых было продемонстрировано, что хорошо известные эффекты и зависимости для однозонных $s$-волновых сверхпроводников эффекты и зависимости при переходе к многозонным сверхпроводникам существенно видоизменяются [3-7]. Поэтому для их объяснения требуется создание и разработка новых теоретических концепций. Одним из ключевых вопросов теории является выявление типа симметрии параметра порядка механизма спаривания куперовских пар в таких сверхпроводниках.

Основные методы исследования сверхпроводящего состояния базируются на изучении термодинамических, транспортных, магнитных или СВЧ свойств сверхпроводника [8-12]. С помощью этих методов могут быть определены такие фундаментальные параметры сверхпроводника, как критический ток, критическая температура, критические магнитные поля, длина когерентности, величина сверхпроводящей щели и др. В то же время изучение зависимостей различных измеряемых величин от температуры, магнитного поля, тока или интенсивности излучения позволяет сделать выводы о природе сверхпроводящего состояния. Для решения актуальных задач требуется создание новых методов исследования сверхпроводящего состояния. Одним из таких перспективных методов является метод ближнепольной СВЧ-микроскопии [13-16]. Преимущество данной методики заключается в возможности локальной и бесконтактной диагностики наличия нескольких энергетических щелей и необычной симметрии параметра порядка в сверхпроводнике.

Первое теоретическое рассмотрение нелинейных СВЧ-эффектов однородных сверхпроводников было выполнено Горьковым и Элиашбергом [17] в рамках нестационарных уравнений Гинзбурга-Ландау для бесщелевого сверхпроводника. Затем в рамках этой модели и квазиклассической теории было исследовано влияние примесей на нелинейный СВЧ-отклик сверхпроводника $[18,19]$. Для высокотемпературных сверхпроводников было показано, что немонотонная зависимость нелинейности в области низких температур может свидетельствовать, например, о $d$-симметрии параметра порядка или о многозонной сверхпроводимости [20-22]. Для описания нелинейных СВЧ-свойств неоднородных по структуре образцов была предложена модель джозефсоновских (слабых) связей [23]. Кроме этого, было предложено большое количество механизмов нелинейности, описывающих поведение вихрей в сверхпроводнике [24]. Также было установлено, что нагрев сверхпроводников может являться существенным при высоких СВЧ-мощностях и даже приводить к разрушению СВЧ-устройств $[25,26]$. Хотя в случае локального нагрева поля, при которых проявляются нелинейные 
эффекты, могут быть значительно уменьшены. Однако, несмотря на большое количество экспериментальных и теоретических работ посвященных этой проблеме, вопрос о механизмах нелинейности в высокотемпературных сверхпроводниках остается актуальным до сих пор. Это связано с тем, что нелинейные СВЧ-свойства несут информацию о природе сверхпроводящего состояния.

В данной работе были синтезированы ВТСП-монокристаллы $(\mathrm{Na}, \mathrm{K})_{x} \mathrm{Fe}_{2-y} \mathrm{Se}_{2}$ с размерами $3 \times 3 \times 0.1 \mathrm{~mm}$. Нелинейные СВЧ-свойства высокотемпературных сверхпроводников исследованы с помощью метода ближнепольной СВЧ-микроскопии. Измерения магнитных свойств монокристаллов $(\mathrm{Na}, \mathrm{K})_{x} \mathrm{Fe}_{2-y} \mathrm{Se}_{2}$ на более низких частотах $(100 \mathrm{kHz})$ были выполнены с помощью стандартного индуктивного метода исследования. При низких температурах обнаружена немонотонная температурная зависимость нелинейного СВЧ-отклика, связанная, по-видимому, с наличием двух щелей в сверхпроводнике.

\section{2. Синтез монокристаллов $(\mathrm{Na}, \mathrm{K})_{x} \mathrm{Fe}_{2-y} \mathrm{Se}_{2}$}

В настоящей работе были исследованы монокристаллы ферроселенида натрия-калия $\left(\mathrm{Na}_{0.3} \mathrm{~K}_{0.7}\right)_{x} \mathrm{Fe}_{2-y} \mathrm{Se}_{2}$ с размерами $3 \times 3 \times 0.1 \mathrm{~mm}$, синтезированные по методике, описанной в [12]. На рис. 1 показано изображение монокристалла $\left(\mathrm{Na}_{0.3} \mathrm{~K}_{0.7}\right)_{x} \mathrm{Fe}_{2-y} \mathrm{Se}_{2}$, полученное с помощью электронного микроскопа. Все этапы приготовления реакционных смесей были выполнены в перчаточном боксе, заполненном аргоном с содержанием $\mathrm{O}_{2}$ и $\mathrm{H}_{2} \mathrm{O}$ менее $0.1 \mathrm{ppm}$. На первой стадии нагреванием смеси порошков $\mathrm{Fe}(99.99 \%)$ и $\mathrm{Se}(99.9 \%)$ при температуре $780^{\circ} \mathrm{C}$ в вакуумированной кварцевой ампуле в течение $48 \mathrm{~h}$ был получен селенид железа $\mathrm{Fe}_{1.02} \mathrm{Se}$. Далее были приготовлены поликристаллические образцы номинального состава $A_{0.8}\left(\mathrm{Fe}_{1.02} \mathrm{Se}\right)_{2}$, где $A=\mathrm{Na}$, К. Для этого кусочки К (99.9\%) или $\mathrm{Na}(99.8 \%)$ смешивали с порошкообразным $\mathrm{Fe}_{1.02} \mathrm{Se}$ в соотношении $A: \mathrm{Fe}_{1.02} \mathrm{Se}=0.8: 2$ и нагревали приготовленные смеси в вакуумированных и запаянных кварцевых ампулах при температуре $380^{\circ} \mathrm{C}$ в течение $6 \mathrm{~h}$. Монокристаллы $\left(\mathrm{K}_{0.7} \mathrm{Na}_{0.3}\right)_{x} \mathrm{Fe}_{2-y} \mathrm{Se}_{2}$ выращивали методом кристаллизации из расплава собственных компонентов (self-flux). Реакционную смесь, состоящую из $\mathrm{Na}_{0.8}\left(\mathrm{Fe}_{1.02} \mathrm{Se}\right)_{2}$ и $\mathrm{K}_{0.8}\left(\mathrm{Fe}_{1.02} \mathrm{Se}\right)_{2}$ в молярном соотношении $3: 7$, помещали в тигель из оксида алюминия и запаивали в небольшую вакуумированную кварцевую ампулу, которую, в свою очередь, помещали в кварцевую ампулу большего диаметра, вакуумировали и запаивали. Приготовленную таким образом двойную кварцевую ампулу с реакционной смесью выдерживали при температуре $1030^{\circ} \mathrm{C}$ в течение $2 \mathrm{~h}$, медленно охлаждали до $700^{\circ} \mathrm{C}$ со скоростью $6^{\circ} \mathrm{C} / \mathrm{h}$, после чего закаливали в воду. Чистота полученных образцов и однородное распределение щелочных металлов

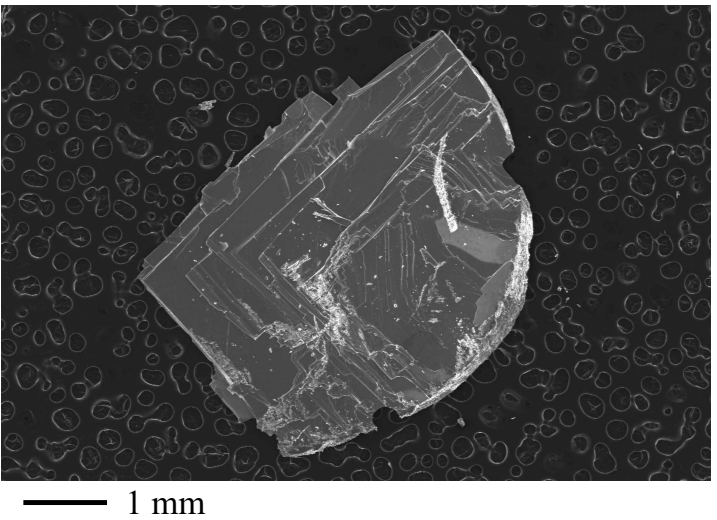

Рис. 1. Изображение монокристалла $(\mathrm{Na}, \mathrm{K})_{x} \mathrm{Fe}_{2-y} \mathrm{Se}_{2}$, полученное с помощью электронного микроскопа.

были подтверждены методами порошковой рентгеновской дифракции и рентгеноспектрального микроанализа.

\section{3. Экспериментальные методики}

В работе были исследованы сверхпроводящие свойства монокристаллов $\left(\mathrm{Na}_{0.3} \mathrm{~K}_{0.7}\right)_{x} \mathrm{Fe}_{2-y} \mathrm{Se}_{2}$ с помощью двух независимых методик. Измерения магнитных свойств монокристаллов на частотах $100 \mathrm{kHz}$ были выполнены с помощью стандартного индуктивного метода исследования [8]. Этот метод основан на эффекте экранирования магнитного поля сверхпроводником (рис. $2, a$ ). В этом случае катушка диаметром $2 \mathrm{~mm}$ по-

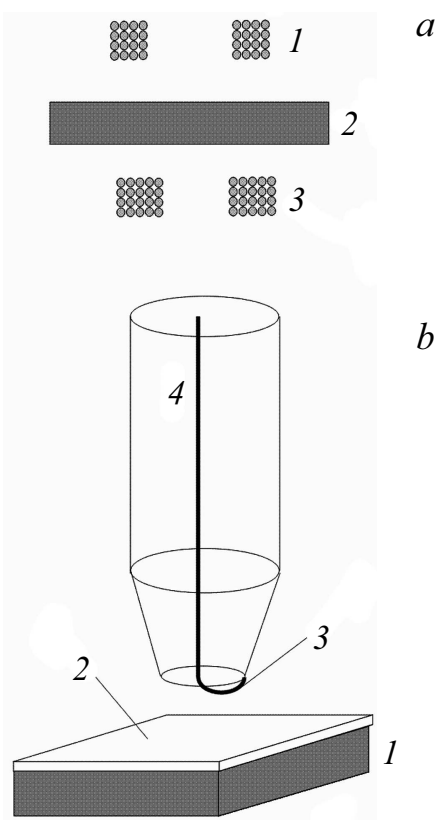

Рис. 2. a) блок-схема зонда для измерений восприимчивости монокристаллов. 1 - передающая катушка, 2 - монокристалл ВТСП, 3 - приемная катушка; $b$ ) блок схема ближнепольного СВЧ зонда. 1 - ВТСП монокристалл, 2 - тефлоновая пленка, 3 - ближнепольный СВЧ зонд, 4 - коаксиальный кабель. 
мещалась с одной стороны пленки и создавала переменное магнитное поле на частоте $100 \mathrm{kHz}$. С помощью второй катушки с другой стороны пленки принимался сигнал на той же частоте. С помощью этого метода магнитная восприимчивость монокристаллов $\chi_{\omega}(T)$ на частоте первой гармоники измерялась в зависимости от температуры и амплитуды переменного магнитного поля.

Второй метод ближнепольной СВЧ-микроскопии основан на регистрации нелинейного СВЧ-отклика с помощью зонда индуктивного типа (рис. 2,b). Зонд представляет собой тонкую медную проволочку закорачивающую внутренний и внешний проводники коаксиального кабеля. Частота первой гармоники в эксперименте была равна $472 \mathrm{MHz}$. Максимальный уровень падающей мощности на частоте первой гармоники при измерениях был порядка $100 \mathrm{~mW}$. C помощью данного метода мощность отраженного сигнала на частоте третьей гармоники $P_{3 \omega}$ измерялась в зависимости от температуры и мощности СВЧ-сигнала. Также следует отметить, что перед измерениями для получения ровной поверхности монокристалла производился скол поверхности образца с помощью скотча. Для предотвращения контактной нелинейности между образцом и ближнепольным СВЧ-зондом помещалась тефлоновая пленка. Более подробно метод ближнепольной СВЧ-микроскопии описан в $[13,14]$.

\section{4. Экспериментальные результаты}

На рис. 3 показана температурная зависимость магнитной восприимчивости монокристалла $\left(\mathrm{Na}_{0.3} \mathrm{~K}_{0.7}\right)_{x} \mathrm{Fe}_{2-y} \mathrm{Se}_{2}$ на частоте первой гармоники $\chi_{\omega}(T)$ при различных амплитудах переменного магнитного поля. Как видно из рис. 3, критическая температура монокристаллов $\left(\mathrm{Na}_{0.3} \mathrm{~K}_{0.7}\right)_{x} \mathrm{Fe}_{2-y} \mathrm{Se}_{2}$ составляет величину $T_{c} \cong 30 \mathrm{~K}$, что согласуется с литературными данными [12]. Также следует отметить, что температурная зависимость $\chi_{\omega}(T)$ для монокристалла

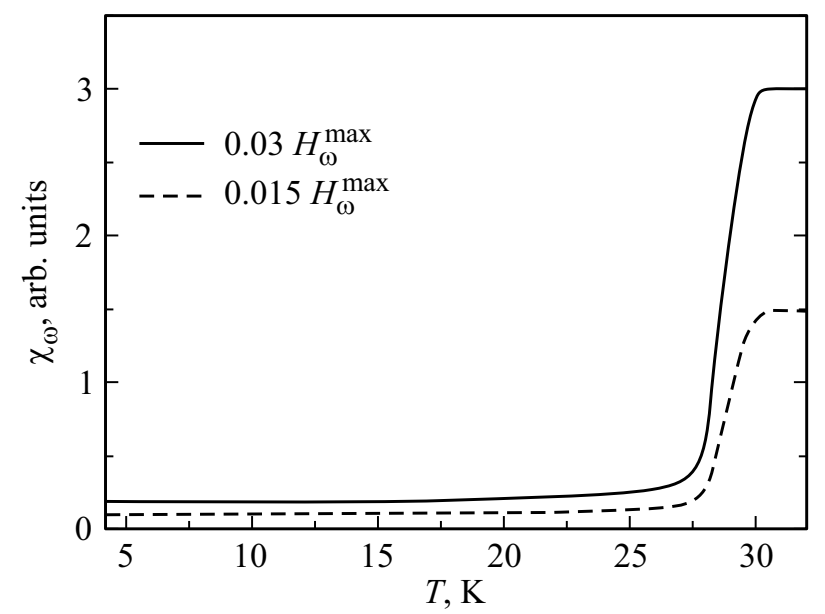

Рис. 3. Температурная зависимость магнитной восприимчивости $\chi_{\omega}(T)$ для монокристалла $(\mathrm{Na}, \mathrm{K})_{x} \mathrm{Fe}_{2-y} \mathrm{Se}_{2}$ при различных амплитудах переменного магнитного поля.

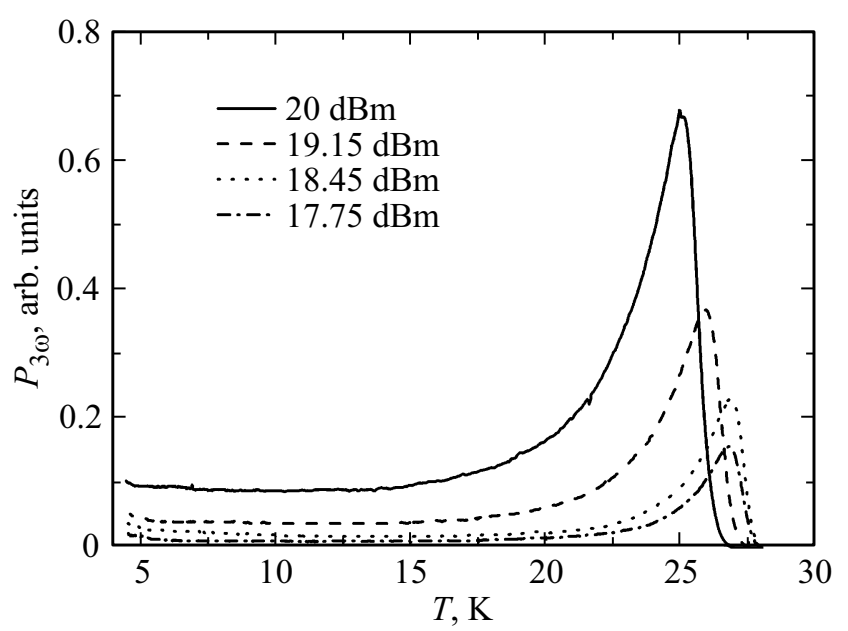

Рис. 4. Температурная зависимость мощности третьей гармоники $P_{3 \omega}(T)$ для монокристалла $(\mathrm{Na}, \mathrm{K})_{x} \mathrm{Fe}_{2-y} \mathrm{Se}_{2}$ при различных СВЧ-мощностях в диапазоне температур от $4.2 \mathrm{~K}$ до критической температуры $T_{c}$.

халькогенида железа демонстрирует один однородный сверхпроводящий переход при различных амплитудах переменного магнитного поля.

На рис. 4 показаны температурные зависимости мощности третьей гармоники $P_{3 \omega}(T)$ для монокристалла $\left(\mathrm{Na}_{0.3} \mathrm{~K}_{0.7}\right)_{x} \mathrm{Fe}_{2-y} \mathrm{Se}_{2}$ при различных СВЧ-мощностях сигнала в диапазоне температур от $4.2 \mathrm{~K}$ до $T_{c}$. Ранее было показано, что для различных сверхпроводников температурная зависимость $P_{3 \omega}(T)$ имеет ярко выраженный максимум, который появляется, когда удельное сопротивление образца обращается в нуль $[13,14]$. Поэтому с помощью метода ближнепольной СВЧ-микроскопии можно бесконтактно определить локальную критическую температуру для монокристалла $T_{c}^{l o c} \cong 27 \mathrm{~K}$. Как и следовало ожидать, критические температуры, определенные с помощью двух методик близки между собой. Из рис. 4 также видно, что температура появления нелинейного СВЧ-отклика немного уменьшается при больших амплитудах падающей СВЧ-мощности. Это связано, по-видимому, с небольшим нагревом образца СВЧ-зондом.

На рис. 5 показаны температурные зависимости мощности третьей гармоники $P_{3 \omega}(T)$ для монокристалла $\left(\mathrm{Na}_{0.3} \mathrm{~K}_{0.7}\right)_{x} \mathrm{Fe}_{2-y} \mathrm{Se}_{2}$ при различных СВЧ-мощностях сигнала при низких температурах. Следует отметить, что поведение температурной зависимости мощности третьей гармоники для монокристаллов $\left(\mathrm{Na}_{0.3} \mathrm{~K}_{0.7}\right)_{x} \mathrm{Fe}_{2-y} \mathrm{Se}_{2}$ качественно отличается от низкотемпературных сверхпроводников [13,14]. В частности, во-первых, из рис. 5 видно, что в области средних температур $P_{3 \omega}(T)$ имеет вид плато, т.е. нелинейный СВЧ-отклик практически не зависит от температуры. Во-вторых, при уменьшении температуры от 10 до $4.2 \mathrm{~K}$ мощность третьей гармоники немного увеличивается. Кроме этого, наблюдается небольшой сдвиг по темпера- 


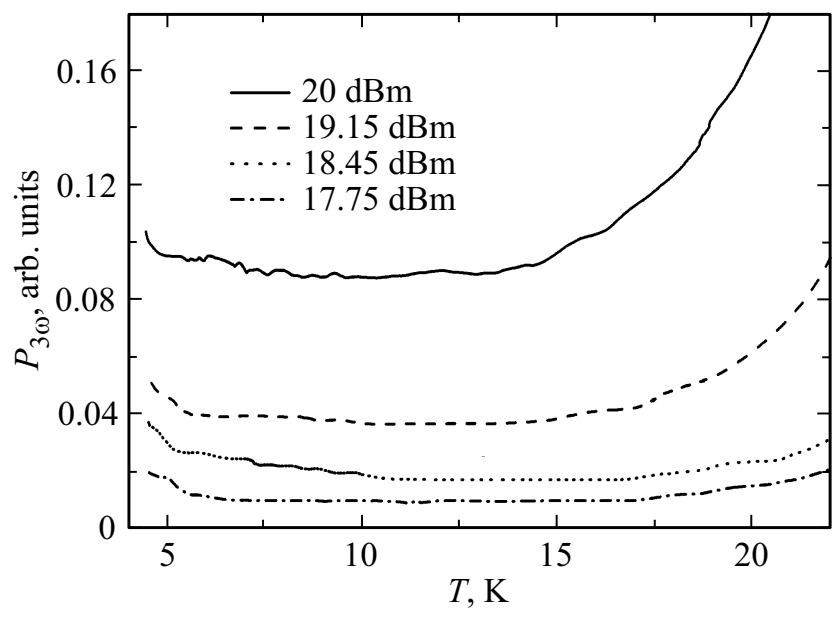

Рис. 5. Температурная зависимость мощности третьей гармоники $P_{3 \omega}(T)$ для монокристалла $(\mathrm{Na}, \mathrm{K})_{x} \mathrm{Fe}_{2-y} \mathrm{Se}_{2}$ при различных СВЧ-мощностях в области низких температур.



Pис. 6. Температурная зависимость мощности третьей гармоники $P_{3 \omega}(T)$ в другом положении СВЧ-зонда на поверхности монокристалла $(\mathrm{Na}, \mathrm{K})_{x} \mathrm{Fe}_{2-y} \mathrm{Se}_{2}$.

туре $P_{3 \omega}(T)$ в зависимости от положения зонда на образце, и в некоторых точках монокристалла температурная зависимость нелинейного СВЧ-отклика демонстрирует второй низкотемпературный пик при температуре примерно $6.5 \mathrm{~K}$ (рис. 6). Следует отметить, что небольшие отличия температурных зависимостей $P_{3 \omega}(T)$ в различных точках на поверхности образца (рис. 5 и 6) связаны, по-видимому, с незначительной неоднородностью состава монокристаллов $\left(\mathrm{Na}_{0.3} \mathrm{~K}_{0.7}\right)_{x} \mathrm{Fe}_{2-y} \mathrm{Se}_{2}$.

Из теории известно, что в приближении слабого сигнала, т. е. когда плотность тока $j$ много меньше плотности критического тока $j_{c}$, связь напряженности электрического поля $E$ с плотностью тока $j$ может быть записана в следующем виде: $E(j)=\alpha(T) j\left(1+b(T) j^{2}\right)$, где $b(T)$ - коэффициент нелинейности. Ранее в работе [22] для двухщелевого сверхпроводника было теоретически показано, что при температурах ниже $0.3 T_{c}$ наблюдает- ся небольшой максимум на температурной зависимости коэффициента нелинейности $b(T)$. Поэтому, поскольку мощность третьей гармоники пропорциональна квадрату коэффициента нелинейности, $P_{3 \omega} \sim b^{2}(T)$, мы считаем, что плато на $P_{3 \omega}(T)$ и наличие второго пика нелинейности обусловлено появлением второй щели в энергетическом спектре монокристалла $\left(\mathrm{Na}_{0.3} \mathrm{~K}_{0.7}\right)_{x} \mathrm{Fe}_{2-y} \mathrm{Se}_{2}$ в области низких температур (рис. 5 и рис. 6). Следует отметить, что наш вывод также согласуется с результатами, полученными ранее авторами работы [12] методом андреевской спектроскопии для монокристаллов $\left(\mathrm{Na}_{0.3} \mathrm{~K}_{0.7}\right)_{x} \mathrm{Fe}_{2-y} \mathrm{Se}_{2}$. В частности, в работе для этого соединения было обнаружено наличие двух сверхпроводящих щелей величиной $\Delta_{L} \cong 9 \mathrm{meV}$ и $\Delta_{S} \cong 2 \mathrm{meV}$.

$\mathrm{C}$ другой стороны, следует отметить, что появление двух максимумов на температурной зависимости $P_{3 \omega}(T)$ может быть также связано с наличием дополнительной сверхпроводящей фазы в монокристалле, имеющей более низкую критическую температуру перехода. Однако, как было отмечено выше, температурная зависимость магнитной восприимчивости $\chi_{\omega}(T)$ демонстрирует однородный переход вблизи критической температуры и не содержит дополнительных особенностей в области низких температур (рис. 3). Поэтому мы считаем, что наличие второго низкотемпературного пика на $P_{3 \omega}(T)$ (рис. 6) не связано с наличием дополнительной сверхпроводящей фазы в монокристалле $\left(\mathrm{Na}_{0.3} \mathrm{~K}_{0.7}\right)_{x} \mathrm{Fe}_{2-y} \mathrm{Se}_{2}$.

На рис. 7 показаны температурные зависимости мощности третьей гармоники $P_{3 \omega}(T)$ для монокристалла $\left(\mathrm{Na}_{0.3} \mathrm{~K}_{0.7}\right)_{x} \mathrm{Fe}_{2-y} \mathrm{Se}_{2}$ в нулевом и в остаточном магнитном поле, перпендикулярном плоскости образца. Из рис. 7 видно, что при включении магнитного поля до величины $250 \mathrm{Oe}$ и его уменьшении до нуля при температуре $4.2 \mathrm{~K}$ температурная зависимость $P_{3 \omega}(T)$ немного сдвигается в сторону более высоких температур. В то же время, при повышении температуры



Рис. 7. Температурная зависимость мощности третьей гармоники $P_{3 \omega}(T)$ для монокристалла $(\mathrm{Na}, \mathrm{K})_{x} \mathrm{Fe}_{2-y} \mathrm{Se}_{2}$ в нулевом магнитном поле (уменьшение температуры) и в остаточном магнитном поле (увеличение температуры). 
выше $T_{c}$ температурная зависимость $P_{3 \omega}(T \mathrm{~F})$ в нулевом магнитном поле полностью повторяется. Поэтому мы считаем, что влияние постоянного магнитного поля на $P_{3 \omega}(T)$, по-видимому, обусловлено наличием запиннингованных вихрей, созданных постоянным магнитным полем.

\section{5. Заключение}

В заключении, в работе методом кристаллизации из расплава собственных компонентов были синтезированы монокристаллы $\left(\mathrm{Na}_{0.3} \mathrm{~K}_{0.7}\right)_{x} \mathrm{Fe}_{2-y} \mathrm{Se}_{2}$ с размерами $3 \times 3 \times 0.1 \mathrm{~mm}$. С помощью метода ближнепольной СВЧ-микроскопии исследованы их нелинейные свойства. В отличие от низкотемпературных сверхпроводников, в халькогенидах железа обнаружено, во-первых, что в области средних температур мощность третьей гармоники практически не зависит от температуры. Вовторых, в диапазоне низких температур в монокристаллах $\left(\mathrm{Na}_{0.3} \mathrm{~K}_{0.7}\right)_{x} \mathrm{Fe}_{2-y} \mathrm{Se}_{2}$ наблюдается второй максимум нелинейности. Наличие этих особенностей на температурной зависимости нелинейного СВЧ-отклика связано, по нашему мнению, с двухщелевой структурой сверхпроводящего состояния в халькогенидах железа. В то же время следует отметить, что для монокристаллов наблюдается влияние внешнего постоянного магнитного поля на температурную зависимость нелинейного СВЧ-отклика монокристаллов. Полученные результаты показывают возможность метода ближнепольной СВЧ-микроскопии локальной и бесконтактной диагностики наличия нескольких энергетических щелей в сверхпроводнике.

Авторы благодарны А.С. Мельникову за полезные замечания при подготовке статьи и А.М. Клушину за помощь в проведении эксперимента.

\section{Список литературы}

[1] J. Nagamatsu, N. Nakagawa, T. Muranaka, Y. Zenitani, J. Akimitsu. Nature 410, 63 (2001).

[2] Y. Kamihara, T. Watanabe, M. Hirano, H. Hosono. J. Am. Chem. Soc. 130, 3296 (2008).

[3] Z. Ren, G. Che, X. Dong, J. Yang, W. Lu, W. Yi, X. Shen, Z. Li, L. Sun, F. Zhou, Z. Zhao. EPL 83, 17002 (2008).

[4] J. Ge, Z. Liu, C. Liu, C. Gao, D. Qian, Q. Xue, Y. Liu, J. Jia. Nature Mater. 14, 285 (2015).

[5] I.I. Mazin, D.J. Singh, M.D. Johannes, M.H. Du. Phys. Rev. Lett. 101, 057003 (2008).

[6] Y. Tanaka. Supercond. Sci. Technol. 28, 034002 (2015); Y.S. Yerin, A.N. Omelyanchouk. Low Temp. Phys. 43, 1263 (2017).

[7] S. Lin. J. Phys.: Condens. Matter 26, 493202 (2014).

[8] J.H. Claassen, M.E. Reeves, R.J. Soulen. Rev. Sci. Instrum. 62, 996 (1991).

[9] М.Р. Трунин. УФН 168, 931 (1998).

[10] Т.Е. Кузьмичёва, С.А. Кузьмичёв, М.Г. Михеев, Я.Г. Пономарёв, С.Н. Чесноков, В.М. Пудалов, Е.П. Хлыбов, Н.Д. Жигадло. УФН 184, 888 (2014).
[11] G. Mu, X.Y. Zhu, L. Fang, L. Shan, C. Ren, H.H. Wen. Chin. Phys. Lett. 25, 2221 (2008).

[12] M. Roslova, S. Kuzmichev, T. Kuzmicheva, Y. Ovchenkov, M. Liu, I. Morozov, A. Boltalin, A. Shevelkov, D. Chareev, A. Vasiliev. Cryst. Eng. Commun. 16, 6919 (2014).

[13] E.E. Pestov, Yu.N. Nozdrin, V.V. Kurin. IEEE Trans. Appl. Supercond. 11, 131 (2001).

[14] А.Ю. Аладышкин, А.А. Андронов, Е.Е. Пестов, Ю.Н. Ноздрин, В.В. Курин, А.М. Cucolo, R. Monaco, М. Boffa. Изв. вуз. Радиофизика 46, 123 (2003).

[15] S.M. Anlage, D.E. Steinhauer, C.P. Vlahacos, B.J. Feenstra, A.S. Thanawalla, W. Hu, S.K. Dutta, F.C. Wellstood. IEEE Trans. Appl. Supercond. 9, 4127 (1999).

[16] T. Tai, B.G. Ghamsari, J.H. Kang, S. Lee, C.B. Eom, S.M. Anlage. Physica C 532, 44 (2017).

[17] Л.П. Горьков, Г.М. Элиашберг. ЖЭТФ 54, 612 (1968).

[18] O. Entin-Wohlman. Phys. Rev. B 18, 4762 (1978).

[19] T. Jujo. J. Phys. Soc. Jpn. 87, 024704 (2018).

[20] B.A. Willemsen, K.E. Kihlstrom, T. Dahm, D.J. Scalapino, B. Gowe, D.A. Bonn, W.N. Hardy. Phys. Rev. B 58, 6650 (1998).

[21] В.В. Курин, А.А. Уткин. ЖЭТФ 127, 652 (2005).

[22] T. Dahm, D.J. Scalapino. Appl. Phys. Lett. 85, 4436 (2004).

[23] J. Halbritter. J. Supercond. 8, 691 (1995).

[24] А.И. Ларкин, Ю.Н. Овчинников. ЖЭТФ 68, 1915 (1975).

[25] M.A. Hein. High-Temperature-Superconductor Thin Films at Microwave Frequencies. Springer, Berlin (1999). 394 p.

[26] T.M. Mishonov, N. Cheenne, D. Robbes, J.O. Indekeu. Eur. Phys. J. B 26, 291 (2002).

Редактор Е.Ю. Флегонтова 